\title{
Evaluation of Treatment Response and Side Effects of Spinal-Z in Patients with Metastatic Gastroesophageal Adenocarcinoma: A Double-Blind Randomized Controlled Trial
}

\author{
Banafsheh Mashak, ${ }^{1}$ Mehran Hoseinzadeh, ${ }^{2}$ Ali Ehsanpour, ${ }^{3}$ Ali Reza Ghanbaran, ${ }^{4,}{ }^{*}$ and Mahdis Vakili ${ }^{5}$ \\ ${ }^{1}$ Department of Anesthesiology, Kemali Hospital, Alborz University of Medical Sciences, Karaj, Iran \\ ${ }^{2}$ Department of Hematology and Oncology, Shafa Hospital, Ahvaz Jundishapur University of Medical Sciences, Ahvaz, Iran \\ ${ }^{3}$ Department of Internal Medicine, Razi Hospital, Ahvaz Jundishpur University of Medical Sciences, Ahvaz, Iran. \\ ${ }^{4}$ Department of Internal Medicine, Imam Khomeini Hospital, Ahvaz Jundishpur University of Medical Sciences, Ahvaz, Iran. \\ ${ }^{5}$ Department of Nutrition, Paramedicine School, Ahvaz Jundishpur University of Medical Sciences, Ahvaz, Iran \\ "Corresponding author: Ali Reza Ghanbaran, Department of Internal Medicine, Imam Khomeini Hospital, Ahvaz Jundishpur University of Medical Sciences, Ahvaz, Iran. Tel: \\ +98-9121467103, Fax: +98-6133367543, E-mail: alirezaghanbaran@yahoo.com
}

Received 2016 November 07; Revised 2016 December 19; Accepted 2017 February 09.

\begin{abstract}
Background: Spinal-Z is a methanolic mixture of dried seed powder of Peganum harmala Linn. and leaf of Dracocephalum kotschyi Boiss. It is available as oral capsules and has been used to treat various cancers. The aim of this study was to evaluate the effects of Spinal-Z capsules (containing herbal extracts) on blood parameters and gastrointestinal symptoms of patients with metastatic gastric adenocarcinoma and to determine its potential side effects.

Methods: A total of 76 patients were randomly assigned into 2 groups. The intervention group received 6 Spinal-Z capsules per day ( 2 after breakfast, 2 after lunch, and 2 after dinner). The control group received placebo capsules in the same manner. The patients were followed-up for 24 weeks.

Results: The distribution of demographic and clinical characteristics was homogeneous in the intervention and control groups. Spinal-Z treatment had no significant effects on cardiovascular parameters, such as systolic blood pressure, diastolic blood pressure, or heart rate, compared to the control group. No significant changes were observed over time, although a significant reduction in fasting blood sugar was observed in the intervention group, compared to the controls. Based on the findings, treatment could significantly reduce dysphagia and stomachache in the follow-up, while it had no significant effects on symptoms, such as itching, nausea, or vomiting neither temporarily nor in the long run (follow-up; $\mathrm{P}>0.05$ ).

Conclusions: Spinal-Z does not have any adverse effects on cardiovascular, renal, and hematopoietic systems or blood glucose level. On the other hand, it could improve gastrointestinal complications. Overall, Spinal-Z, as an accessible and affordable medication with very few side effects, may be a suitable option for treating complications in patients with metastatic gastroesophageal adenocarcinoma, as it can decrease fasting blood sugar and erythrocyte sedimentation rate.
\end{abstract}

Keywords: Cancers, Spinal-Z, Peganum harmala Linn

\section{Background}

The prevalence of gastric adenocarcinoma is rising worldwide. This disease is recognized as the fourth most prevalent cancer and the third cause of mortality worldwide (1). In developing countries, the cumulative risk has been estimated at 1.1\%, and the associated mortality rate is as high as $0.9 \%$. As $70 \%$ of all new cases and deaths of gastric adenocarcinoma are reported in developing countries (2), prevention and treatment are essential in countries such as Iran.

Complementary and alternative medicine (CAM) provides treatment options for gastric cancer. Based on a recent meta-analysis, CAM treatments have grown in popularity in many countries (3). One of the herbal treatments for gastric cancer complications in Iran is Spinal-Z. This medication is a methanolic mixture of dried seed powder of Peganum harmala Linn and leaf of Dracocephalum kotschyi Boiss. It is available as edible capsules and has been used to treat various cancers. According to the literature, this herbal medicine can reduce the viability of cancerous cell lines in mice (4).

Peganum harmala is one of the traditional treatments for various diseases including cancer. Based on previous reports, alkaloids found in this plant can prevent tumor cell growth. This plant is an herbaceous perennial plant of the family Zygophyllaceae, native to different regions, including countries of the Middle East such as Iran $(4,5)$. It can also improve cell lysis within 24 hours after use (6).

Traditionally, Dracocephalum kotschyi has been used independently as a treatment for rheumatic diseases due to

Copyright (c) 2017, Jundishapur Journal of Chronic Disease Care. This is an open-access article distributed under the terms of the Creative Commons 
its inhibitory effects on lectin-induced cellular immune responses. Moreover, another component of Spinal-Z, calycopterin, can inhibit mitogen-induced lymphocyte proliferation (7). Calycopterin can also induce anticancer effects due to the involvement of P-CREB and phase-II detoxifying enzyme system (8). Xanthomicrol is also one of the main cytotoxic components of Dracocephalum kotschyi and a potential anticancer agent (4).

Considering the molecular structure of Spinal-Z, this physiologically compatible agent has no major side effects (4). Moreover, it has cytotoxic, antiinflammatory, antiedema, pain relief, antibacterial, and antiviral effects (6). With this background in mind, the aim of the present study was to evaluate the effect of Spinal-Z on gastrointestinal symptoms of patients with gastric adenocarcinoma.

\section{Methods}

In this double-blind randomized controlled trial, 76 patients with gastric adenocarcinoma, admitted to Shafa Hospital (a teaching hospital), were allocated to the intervention and control groups. The inclusion criteria were as follows:1) metastatic phase of cancer (stage IV);2) no use of chemotherapy drugs during the study; and 3) diagnosis of gastric adenocarcinoma. On the other hand, the exclusion criteria were serum creatinine level $>2 \mathrm{mg} / \mathrm{dL}$ and ejection fraction $<45 \%$.

A total of 76 patients were randomly assigned into the intervention and control groups (n, 36 per group). The intervention group received 6 Spinal-Z capsules ( 2 after breakfast, 2 after lunch, and 2 after dinner). The control group received placebo capsules in the same manner for 24 weeks.

\subsection{Medications and Measurements}

Spinal-Z was manufactured and formulated by Darupakhsh Co. (Iran) and contained $200 \mathrm{mg}$ of Peganum harmala Linn. (20\%) and Dracocephalum kotschyi Boiss (80\%) powder. The medications were given to the participants free of charge every week, and patients' adherence to the regimen was monitored via phone calls every day in both intervention and control groups.

The following parameters were measured using the participants' blood samples: systolic blood pressure, diastolic blood pressure, heart rate, blood electrolytes including sodium, potassium, and creatinine, fasting blood sugar (FBS) level, erythrocyte sedimentation rate (ESR), liver function enzymes including aspartate aminotransferase (AST) and alanine aminotransferase (ALT), hemoglobin level, white blood cell (WBC) count, platelet count, alkaline phosphatase level, total bilirubin level, and direct bilirubin level. All the measurements were performed at baseline and after 2, 8, 12, 16, and 24 weeks.

\subsection{Ethical Considerations}

All institutional and governmental regulations concerning the ethical use of human volunteers were followed during this research. Written informed consents were obtained from all the participants, and the ethics committee of Ahvaz Jundishapur University of Medical Sciences approved the study (Ref No., U-91175). The study protocol was registered at the Iranian registry of randomized controlled trials (IRCT2013010111967N1).

\subsection{Statistical Analysis}

The significance level was set at a 2-tailed type I error of 0.05. All statistical analyses were performed using SPSS version 22. Categorical data are reported as number (percentage), and continuous variables are presented as mean \pm SD. Shapiro-Wilk test was used to examine the normal distribution of quantitative variables. To compare the demographic characteristics of the groups, independent sample $t$ test or nonparametric Mann-Whitney U test (if needed) was used for continuous variables, and Pearson's Chi square test was conducted for nominal variables.

Generalized estimating equation (GEE) (1) model was applied to examine the differences between the groups (intervention and control groups) in terms of changes in clinical measures (eg, serum creatinine level, FBS, blood pressure, heart rate, hemoglobin level, platelet count, WBC count, and liver enzymes) and side effects (eg, dysphagia, abdominal pain, anorexia, itching, nausea, and vomiting) over time. The GEE model included 2 main effects (group and time), as well as the interactions of these effects. Time points in the analyses included baseline and weeks 2, 4, 8, 12,16 , and 24. P value less than 0.05 was considered statistically significant, and all tests were 2 -sided. SPSS version 18.0.0 (SPSS Inc., Chicago, IL, USA) was used for all statistical analyses.

\section{Results}

\subsection{Participants}

In total, 76 patients were randomized between December 2012 and January 2014. The patients were analyzed in terms of baseline characteristics (Figure 1).

\subsection{Demographic Characteristics}

The mean age of the participants was 56.16 years. Overall, $56.6 \%$ of the subjects were male $(57.9 \%$ of the control group and $55.3 \%$ of the intervention group) and $43.4 \%$ were 


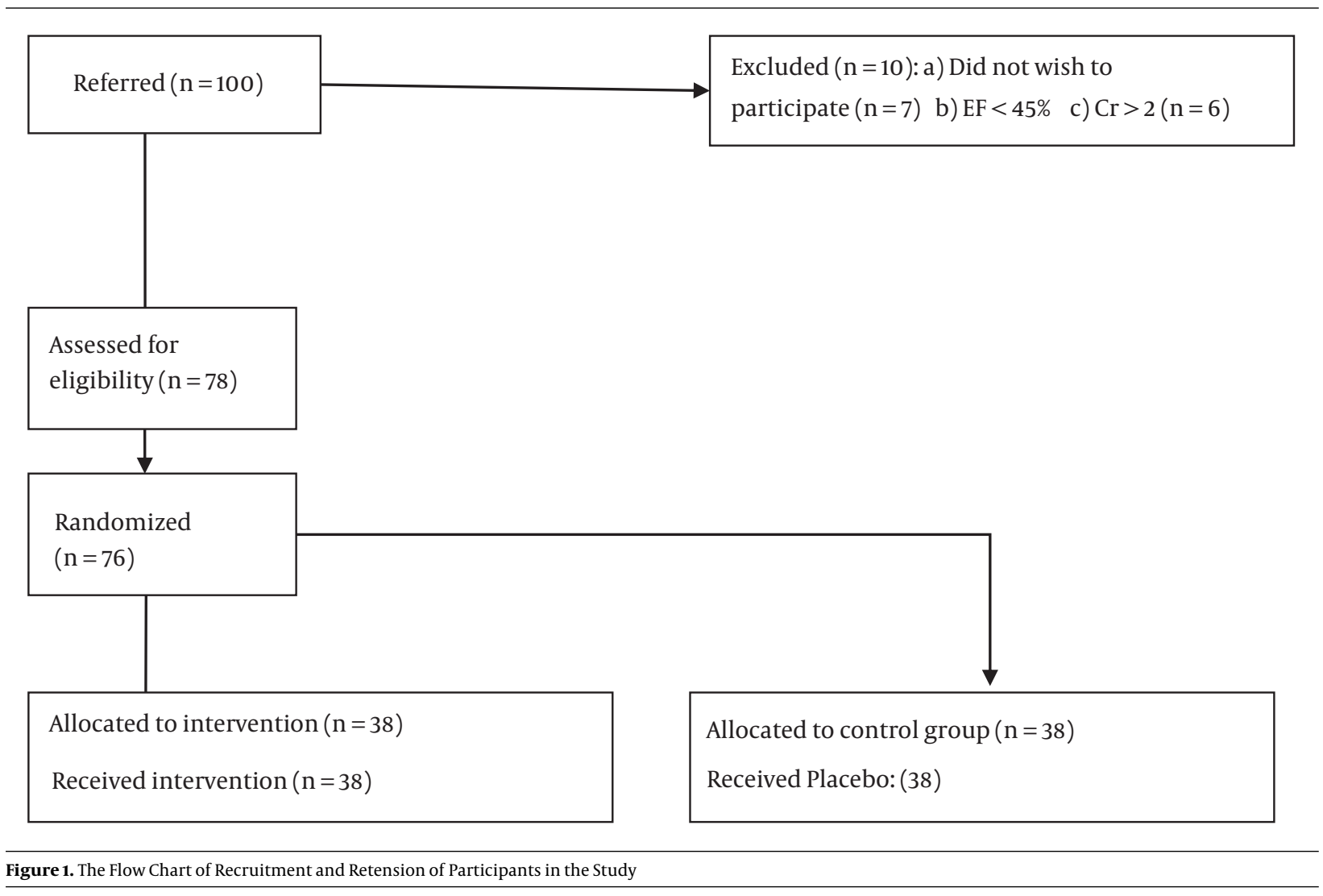

female $(42.1 \%$ of the control group and $44.7 \%$ of the intervention group). The distribution of demographic and clinical characteristics was homogeneous in the intervention and control groups. The demographic characteristics of the participants are presented in Table 1.

\subsection{Spinal-Z and Laboratory Parameters}

The variables in the intervention and control groups were evaluated regarding the effect of time alone and interaction of time and treatment. Spinal-Z treatment had no significant effects on cardiovascular parameters, such as systolic blood pressure, diastolic blood pressure, or heart rate, compared to the control group; time also did not cause any changes in the outcomes. We did not detect any significant counteraction between time and treatment. Similar results were reported for the hematopoietic system (WBC count, platelet count, and hemoglobin level), hepatic factors (AST, ALT, alkaline phosphate, and total bilirubin levels), and blood electrolytes (sodium, potassium, and creatinine levels).

Spinal-Z could significantly decrease blood glucose level during the follow-up ( $\mathrm{P}=0.01)$; however, timetreatment interaction had no significance. We observed a significant decline in ESR in the intervention group during the follow-up $(\mathrm{P}<0.05)$. In addition, a significant interaction between time and treatment was reported $(\mathrm{P}<0.05)$.

\subsection{Spinal-Z and Gastrointestinal Problems}

Spinal-Z could significantly decrease gastrointestinal problems over time $(\mathrm{P}<0.5)$.

\subsection{Side Effects}

Treatment with Spinal-Z did not have any effects on patients' symptoms such as itching, nausea, and vomiting neither temporarily nor in the long run (after 24 weeks of follow-up; P> 0.05).

\section{Discussion}

This randomized controlled clinical trial was performed to evaluate the effects of Spinal-Z, a capsule containing a mixture of herbal extracts, on blood parameters and gastrointestinal symptoms and to study its potential side effects in patients with metastatic gastric adenocarcinoma. Peganum harmala as one of the 2 constituents of Spinal-Z is known to reduce proliferation and affect cell lysis in cancerous cell lines (9). 
Table 1. Demographic Characteristics of the Participants ${ }^{\mathrm{a}}$

\begin{tabular}{|c|c|c|c|}
\hline Variables & Intervention Group, $\mathrm{n}=\mathbf{3 8}$ & Control Group, $n=38$ & PValue $^{\text {b }}$ \\
\hline Age (years) & $57.45 \pm 12.87$ & $56.78 \pm 11.6$ & 0.81 \\
\hline Gender & & & $>0.99$ \\
\hline Female & $17(44.7)$ & $16(42.1)$ & \\
\hline Male & $21(55.3)$ & $22(57.9)$ & \\
\hline
\end{tabular}

${ }^{\mathrm{a}}$ Values are expressed as Mean $\pm \mathrm{SD}$ or $\mathrm{N}(\%)$.

${ }^{\mathrm{b}}$ There was no significant difference between the groups.

Table 2. Blood Parameters in the Intervention and Control Groups During Treatment and Follow-Up ${ }^{\mathrm{a}}$

\begin{tabular}{|c|c|c|c|c|c|c|c|}
\hline & & Week 0 & Week 8 & Week 12 & Week 16 & Week 24 & PValue $^{\text {b }}$ \\
\hline \multirow{2}{*}{ Hemoglobin } & Spinal-Z & $11.4(1.07)$ & $11.4(1.08)$ & & $11.43(0.94)$ & $11.8(1.1)$ & \multirow{2}{*}{0.96} \\
\hline & Placebo & $11.2(1.1)$ & $11.1(1)$ & & $11.2(0.88)$ & $11.2(0.9)$ & \\
\hline \multirow{2}{*}{ WBC } & Spinal-Z & $8080(2210)$ & $8028(1956)$ & & $8014(1770)$ & $8014(1770)$ & \multirow{2}{*}{0.12} \\
\hline & Placebo & $8220(2200)$ & $800(1800)$ & & $7890(1830)$ & $7770(1670)$ & \\
\hline \multirow{2}{*}{ Platelet count } & Spinal-Z & $171.47(3800)$ & $181.18(38.2)$ & & $182.08(93.2)$ & $180.74(38.08)$ & \multirow{2}{*}{0.38} \\
\hline & Placebo & $179.29(37.3)$ & $179.13(35.8)$ & & $183.3(39.8)$ & $179.7(36.5)$ & \\
\hline \multirow{2}{*}{ Systolic blood pressure } & Spinal-Z & $121.9(14.1)$ & $122.7(14.8)$ & & $124.08(19)$ & $122.7(14.9)$ & \multirow{2}{*}{0.97} \\
\hline & Placebo & $120.8(18.8)$ & $120(13.2)$ & & $120.02(15.1)$ & $119.6(15.4)$ & \\
\hline \multirow{2}{*}{ Diastolic blood pressure } & Spinal-Z & $78.6(6.1)$ & $78.9(6.4)$ & & $79.6(5.5)$ & $78.6(4.1)$ & \multirow{2}{*}{0.79} \\
\hline & Placebo & $78.1(6)$ & $77.7(5.5)$ & & $75.9(6.4)$ & $78(4.8)$ & \\
\hline \multirow{2}{*}{ Heart rate } & Spinal-Z & $78.3(7.2)$ & $79.5(6.1)$ & & $79(5.6)$ & $78.3(4.8)$ & \multirow{2}{*}{0.32} \\
\hline & Placebo & $80.6(6.3)$ & $81.4(5.5)$ & & $80.5(4.9)$ & $79.9(4.5)$ & \\
\hline \multirow{2}{*}{ Creatinine } & Spinal-Z & $1.1(0.31)$ & & $1.1(0.3)$ & & $1.11(0.29)$ & \multirow{2}{*}{0.42} \\
\hline & Placebo & $1.1(0.29)$ & & $1.19(0.28)$ & & $1.19(0.29)$ & \\
\hline \multirow{2}{*}{ Calcium } & Spinal-Z & $139.6(2.2)$ & & $139.3(1.6)$ & & $137.03(1.6)$ & \multirow{2}{*}{0.41} \\
\hline & Placebo & $140(2.09)$ & & $139.89(2.9)$ & & $139.6(2.03)$ & \\
\hline \multirow{2}{*}{ Potassium } & Spinal-Z & $4.08(0.16)$ & & $4.05(0.15)$ & & $4.05(0.09)$ & \multirow{2}{*}{0.051} \\
\hline & Placebo & $4.05(0.14)$ & & $4.01(0.08)$ & & $4.09(0.08)$ & \\
\hline \multirow{2}{*}{ ESR } & Spinal-Z & $59.08(20.7)$ & & $53.9(16.6)$ & & $40.7(12.3)$ & \multirow{2}{*}{$<0.05$} \\
\hline & Placebo & $65.16(21.9)$ & & $63.18(19.9)$ & & $62.89(20.9)$ & \\
\hline \multirow{2}{*}{ FBS } & Spinal-Z & $102.9(20.7)$ & $100.3(15.6)$ & & $100.7(16.5)$ & $99.87(16)$ & \multirow{2}{*}{0.78} \\
\hline & Placebo & $97.6(14)$ & $96.4(12.5)$ & & $95.5(13.4)$ & $95(13)$ & \\
\hline \multirow{2}{*}{ AST } & Spinal-Z & $31.5(14.8)$ & & $31.2(13.8)$ & & $33.5(13.5)$ & \multirow{2}{*}{0.3} \\
\hline & Placebo & $28.1(14.2)$ & & $27.4(13.2)$ & & $26.7(13)$ & \\
\hline \multirow{2}{*}{ ALT } & Spinal-Z & $26.1(13.4)$ & & $25.7(13)$ & & $26.5(13)$ & \multirow{2}{*}{0.57} \\
\hline & Placebo & $24(10)$ & & $23.9(10)$ & & $23.7(9.2)$ & \\
\hline \multirow{2}{*}{ Alkaline phosphatase } & Spinal-Z & $228.4(22.5)$ & & $226.6(20.2)$ & & $226.9(18)$ & \multirow{2}{*}{0.44} \\
\hline & Placebo & $231.7(14.2)$ & & $231.1(12.8)$ & & $234.6(35.7)$ & \\
\hline \multirow{2}{*}{ Total bilirubin } & Spinal-Z & $1(0.17)$ & & $1(0.16)$ & & $1.01(0.13)$ & \\
\hline & Placebo & $1.01(0.17)$ & & $0.99(0.15)$ & & $1.01(0.13)$ & 0.87 \\
\hline & Spinal-Z & $0.45(0.09)$ & & $0.45(0.09)$ & & $0.45(0.07)$ & \\
\hline Direct bilirubin & Placebo & $0.44(0.11)$ & & $0.43(0.1)$ & & $0.45(0.09)$ & 0.3 \\
\hline
\end{tabular}

${ }^{\mathrm{a}}$ The values are expressed as mean (SD).

${ }^{\mathrm{b}} \mathrm{P}$ value for group-time interaction based on the results of GEE analysis.

The leaf extract of $D$. kotschyi can inhibit tumor proliferation in mice. Spinal-Z and its constituents can reduce the viability of cells in a dose-dependent manner (4). From a pharmacological viewpoint, Peganum harmala has antibac- 
Table 3. The Incidence of Gastrointestinal Problems During Treatment and Follow-Up in the Intervention and Control Groups (n, 38 Per Group) ${ }^{\mathrm{a}}$

\begin{tabular}{|c|c|c|c|c|c|c|c|}
\hline Gastrointestinal Problems & & Week o & Week 2 & Week 4 & Week 16 & Week 24 & P Value $^{b}$ \\
\hline \multirow{2}{*}{ Nausea and vomiting } & Spinal-Z & 0 & $7(18.4)$ & $6(15.8)$ & 0 & 0 & \multirow{2}{*}{0.22} \\
\hline & Placebo & 0 & 0 & 0 & 0 & 0 & \\
\hline \multirow{2}{*}{ Dysphagia } & Spinal-Z & $24(63.1)$ & $24(63.1)$ & $26(68.4)$ & $14(36.8)$ & $7(18.4)$ & \multirow{2}{*}{$<0.05$} \\
\hline & Placebo & $21(55.2)$ & $21(55.2)$ & $21(55.2)$ & $23(60.5)$ & $24(63.1)$ & \\
\hline \multirow{2}{*}{ Poor appetite } & Spinal-Z & $28(73.6)$ & $33(86.8)$ & $35(92.1)$ & $14(36.8)$ & $10(26.3)$ & \multirow{2}{*}{$<0.05$} \\
\hline & Placebo & $26(68.4)$ & $22(57.8)$ & $15(39.4)$ & $25(65.7)$ & $25(65.7)$ & \\
\hline \multirow{2}{*}{ Stomachache } & Spinal-Z & $35(92.1)$ & $33(86.8)$ & $26(68.4)$ & $8(21.05)$ & $8(21.05)$ & \multirow{2}{*}{$<0.05$} \\
\hline & Placebo & $35(92.1)$ & $35(92.1)$ & $35(92.1)$ & $34(89.4)$ & $34(89.4)$ & \\
\hline \multirow{2}{*}{ Itching } & Spinal-Z & 0 & $3(7.9)$ & $2(5.83)$ & 0 & 0 & \multirow{2}{*}{0.31} \\
\hline & Placebo & & & & & & \\
\hline
\end{tabular}

${ }^{\mathrm{a}}$ The values are expressed as No. (\%).

${ }^{\mathrm{b}} \mathrm{P}$ value for group-time interaction based on the results of GEE analysis.

terial effects against microorganisms such as Staphylococcus aureus, Pseudomonas aeruginosa, Escherichia coli, and Klebsiella pneumoniae (10), as well as ameliorative effects on the cardiovascular system (eg, antispasmodic, anticholinergic, antihistaminic, and antiadrenergic effects) (11). However, in the present study, this agent had no significant effects on the cardiovascular or serological system.

Seeds and roots of Spinal-Z are reported to have antifungal and antileishmanial activities $(12,13)$. In this study, this agent could significantly decrease FBS level (not in comparison to the control group). However, we could not find any similar studies for comparison. Spinal-Z could significantly increase the patients' appetite and reduce dysphagia and stomachache in the follow-up. This finding might be related to the antibacterial or other similar features of this agent on bacteria, fungi, and gut flora. Nevertheless, we could not find any studies regarding the effect of Spinal-Z or its components on gastrointestinal symptoms.

\subsection{Limitations}

Some limitations of the present study should be acknowledged. Spinal-Z is only available in Iran and we could not find any other studies investigating the effects or side effects of this agent. In addition, lifestyle, eating habits, and environmental factors can strongly affect gastrointestinal complications. Therefore, caution should be taken while interpreting the results. Further research with a larger sample size about the effect of Spinal-Z is recommended.

\subsection{Conclusion}

Spinal-Z does not have any adverse effects on cardiovascular, renal, and hematopoietic systems or blood glucose level. Spinal-Z as an accessible and affordable medication with very few side effects may be a suitable option for treating complications in patients with metastatic gastroesophageal adenocarcinoma.

\section{Acknowledgments}

This paper was retrieved from a thesis of MD (project code, D.716). The study was funded by Ahvaz Jundishapur University of Medical Sciences. We would like to thank the staff of Ahvaz Jundishapur University of Medical Sciences for their collaboration. We also express our gratitude to Dr. Parvin Abedi for revising and editing this paper.

\section{Footnote}

Conflicts of Interest: The authors declare no conflicts of interest.

\section{References}

1. Kim J, Fox C, Peng S, Pusung M, Pectasides E, Matthee E, et al. Preexisting oncogenic events impact trastuzumab sensitivity in ERBB2-amplified gastroesophageal adenocarcinoma. J Clin Invest. 2014;124(12):5145-58. doi: 10.1172/JCI75200. [PubMed: 25401468].

2. Jemal A, Bray F, Center MM, Ferlay J, Ward E, Forman D. Global cancer statistics. CA Cancer J Clin. 2011;61(2):69-90. doi: 10.3322/caac.20107. [PubMed: 21296855].

3. Horneber M, Bueschel G, Dennert G, Less D, Ritter E, Zwahlen M. How many cancer patients use complementary and alternative medicine: a systematic review and metaanalysis. Integr Cancer Ther. 2012;11(3):187-203. doi: 10.1177/1534735411423920. [PubMed: 22019489]. 
4. Jahaniani F, Ebrahimi SA, Rahbar-Roshandel N, Mahmoudian M. Xanthomicrol is the main cytotoxic component of Dracocephalum kotschyii and a potential anti-cancer agent. Phytochemistry. 2005;66(13):1581-92. doi: 10.1016/j.phytochem.2005.04.035. [PubMed: 15949825].

5. Lamchouri F, Zemzami M, Jossang A, Abdellatif A, Israili ZH, Lyoussi B. Cytotoxicity of alkaloids isolated from Peganum harmala seeds. PakJ Pharm Sci. 2013;26(4):699-706. [PubMed: 23811445].

6. Lamchouri F, Settaf A, Cherrah Y, Hassar M, Zemzami M, Atif N. In vitro cell-toxicity of Peganum harmala alkaloids on cancerous cell-lines. Fitoterapia. 2000.

7. Faham N, Javidnia K, Bahmani M, Amirghofran Z. Calycopterin, an immunoinhibitory compound from the extract of Dracocephalum kotschyi. Phytother Res. 2008;22(9):1154-8. doi: 10.1002/ptr.2382. [PubMed: 18683896].

8. Fitzmaurice G, Laird N, Ware J. Applied longitudinal analysisle. Wiley.
2011.

9. Sarvestani NN, Khodagholi F, Ansari N, Farimani MM. Involvement of p-CREB and phase II detoxifying enzyme system in neuroprotection mediated by the flavonoid calycopterin isolated from Dracocephalum kotschyi. Phytomedicine. 2013;20(10):939-46.

10. Lamchouri F, Settaf A, Cherrah Y, Hassar M, Zemzami M, Atif N, et al. In vitro cell-toxicity of Peganum harmala alkaloids on cancerous celllines. Fitoterapia. 2000;71(1):50-4. [PubMed:11449470].

11. Prashanth D, John S. Antibacterial activity of Peganum harmala. Fitoterapia. 1999.

12. Aqel M, Hadidi M. Direct Relaxant Effect of Peganum Harmala Seed Extract on Smooth Muscles of Rabbit and Guinea Pig. Int J Pharmacogn. 2008.

13. Saadabi AMA. Antifungal activity of some saudi plants used in traditional medicine. Asian J Plant Sci. 2006 